\title{
El servicio como principio que une y potencia las organizaciones: una revisión y propuesta conceptual en torno a la cultura de servicio
}

\author{
ALFREDO GORROCHOTEGUI-MARTELL ${ }^{a}$, \\ ROSARIO MONTT-RODRÍGUEZ', \\ MAITE NAZARRE-CORDERO'
}

\begin{abstract}
RESUMEN En este artículo se hace una reflexión y una revisión sobre los distintos autores que han expuesto sus conceptos acerca del servicio. Se busca rescatar la idea de que todas las organizaciones ofrecen un servicio a la sociedad. Además el artículo busca aproximarse a la naturaleza del servicio, sus cualidades y sus características; indagar en el servicio como experiencia humana, de cara al cliente y dentro de la empresa; distinguir los conceptos de cliente, cliente interno, momentos de la verdad, ciclos y mapas de servicio, para luego adentrarse en la posibilidad de configurar una auténtica cultura del servicio. Y en todo este desarrollo, se indaga también sobre la posibilidad que tiene el servicio como un principio noble que une y que potencia a las personas que trabajan en organizaciones que poseen una cultura centrada en sus clientes internos y externos.
\end{abstract}

PALABRAS CLAVE cliente interno, cultura del servicio, cultura organizacional, desarrollo organizacional, experiencias de servicio, servicio.

\author{
HISTORIA DEL ARTÍCULO \\ ¿CÓMO CITAR?: \\ Gorrochotegui-Martell, A., Montt- \\ Rodríguez, R. \& Nazarre-Cordero, \\ M. (2015). El servicio como \\ principio que une y potencia las \\ organizaciones: una revisión y \\ propuesta conceptual en torno a \\ la cultura de servicio. Perspectiva \\ Empresarial, 2(2), 55-71. http:// \\ dx.doi.org/10.16967\%2Frpe.v2n2a6 \\ RECIBIDO: 23 de abril de 2015 \\ APROBADO: 14 de septiembre \\ de 2015 \\ CORRESPONDENCIA: \\ Alfredo Gorrochotegui, Universidad \\ de los Andes, Escuela de \\ Administración de Servicios, Av. \\ Monseñor Álvaro del Portillo 12.455 , \\ Las Condes, Codigo Postal 7620001 , \\ Santiago, Chile.
}

\footnotetext{
a Doctor, docente, Universidad de los Andes, Chile. Correo electrónico: agorrochotegui@uandes.cl

b Magíster, docente, Universidad de los Andes, Chile. Correo electrónico: rmontt@uandes.cl

c Licenciada, docente, Universidad de los Andes, Chile. Correo electrónico: mnazarre@uandes.cl
} 


\section{Service as a principle that brings together and boosts organizations: A review and conceptual proposal revolving around service culture}

ABSTRACT This article reflects on and reviews the different authors that have presented their concepts of service. It seeks to recover the idea that all organizations offer a service to society. In addition, the article aims to approach service nature, qualities, and features; inquire into service as a human experience, in regards to the customer, and within the company; distinguish the concepts of customer, internal customer, moments of truth, service cycles and maps, to delve into the possibility of creating an authentic service culture. In all this elaboration, the article also inquiries into the possibility of service as a noble principle that brings together and boosts individuals working in organizations with an internal / external customer-oriented culture.

KEYWORDS internal customer, service culture, organizational culture, organizational development, service experiences, service.

¿CÓMO CITO EL ARTÍ́CULO? HOW TO CITE THIS PAPER?

\section{CHICAGO:}

Gorrochotegui-Martell, Alfredo, Montt-Rodríguez, Rosario y Nazarre-Cordero, Maite. 2015. "El servicio como principio que une y potencia las organizaciones: una revisión y propuesta conceptual en torno a la cultura de servicio". Perspectiva Empresarial 2(2): 55-71. http://dx.doi. org/10.16967\%2Frpe.v2n2a6

MLA:

Gorrochotegui-Martell, Alfredo, Montt-Rodríguez, Rosario y Nazarre-Cordero, Maite. "El servicio como principio que une y potencia las organizaciones: una revisión y propuesta conceptual en torno a la cultura de servicio". Perspectiva Empresarial 2.2 (2015): 55-71. Digital. http://dx.doi. org/10.16967\%2Frpe.v2n2a6

\section{O serviço como princípio que une e potencializa as organizações: uma revisão e proposta conceitual sobre a cultura de serviço}

RESUMO Neste artigo, faz-se uma reflexão e uma revisão sobre os diferentes autores que vêm expondo seus conceitos sobre o serviço. Busca-se resgatar a ideia de que todas as organizações oferecem um serviço à sociedade. Além disso, este artigo procura aproximar-se à natureza do serviço, suas qualidades e suas características; indagar o serviço como experiência humana, voltado ao cliente e dentro da empresa; diferenciar os conceitos de cliente, cliente interno, momentos da verdade, ciclos e mapas de serviço para, em seguida, se aprofundar na possibilidade de configurar uma autêntica cultura do serviço. Em todo esse desenvolvimento, questiona-se também sobre a possibilidade que o serviço tem como um princípio nobre que une e potencializa as pessoas que trabalham em organizações que possuem uma cultura centralizada em seus clientes internos e externos.

PALAVRAS CHAVE cliente interno, cultura do serviço, cultura organizacional, desenvolvimento organizacional, experiências de serviço, serviço. 


\section{Introducción}

El estudio acerca de los servicios ha evolucionado raudamente durante los últimos años, dirigiéndose hacia la gestión de la experiencia que viven los clientes. Este recorrido ha exigido la utilización de herramientas propias del diseño que permitan ejercer un mayor control y desarrollo de los procesos internos. Los autores de este estudio pretenden contextualizar el ambiente de los servicios a partir de lo anteriormente expuesto, con el fin de entregar una visión humana del servicio, que resalta la importancia de las personas que influyen directa o indirectamente en las vivencias del cliente. Aquí se entiende al cliente interno como principio unificador de la experiencia y, a su vez, generador de cultura dentro de las organizaciones. Esta cultura constituye la raíz de lo que se le transmite al cliente a través del servicio; por esta razón, se hace fundamental la creación de una filosofía de servicio que sustente y potencie el quehacer humano dentro de las organizaciones.

A continuación se presenta un diagrama explicativo y organizador de los términos esenciales que se desarrollarán a lo largo de este trabajo (ver figura 1).

\section{Las organizaciones y el servicio}

Para comenzar esta reflexión, una frase de Peter Drucker: "La organización moderna existe para prestar un servicio específico a la sociedad" (1981, p. 509). En otras obras, el autor diferencia unas organizaciones de otras en lo "específico" por alcanzar. Pero su objetivo es hacernos recapacitar sobre una realidad que cada día se ve más clara: todas las organizaciones ofrecen un servicio.

Horovitz (1990, p. xi) afirma que "hoy la ofensiva se centra en el campo de los servicios. Servicios antes, durante y después de la venta de productos, pero servicio también en los sectores de los servicios propiamente dichos: banca, transporte, turismo, servicios públicos, y muchos otros". Lo anterior indica dos aspectos: a) que toda empresa ofrece servicios aunque objetivamente entregue productos de fabricación, y b) que las tradicionalmente llamadas empresas de servicios están observándose a sí mismas para sacar mayor provecho de su propia naturaleza.

Diferenciar una organización de otra en cuanto al producto que ofrece ya no es hoy en día lo importante. Zeithaml, Parasuraman \& Berry (1993, pp. 1-2) exponen que:

Prácticamente todas las organizaciones compiten, de una y otra forma, en el área del servicio. Es difícil mencionar un sector en el que el servicio no sea importante [...] En la medida que los ejecutivos de las organizaciones industriales encuentren más dificultades para crear ventajas tecnológicas competitivas y duraderas, deberán prestar una mayor y más directa atención [...] al valor añadido que representa el servicio como una verdadera fuente de

FIGURA 1. Diagrama explicativo de los conceptos esenciales de este trabajo

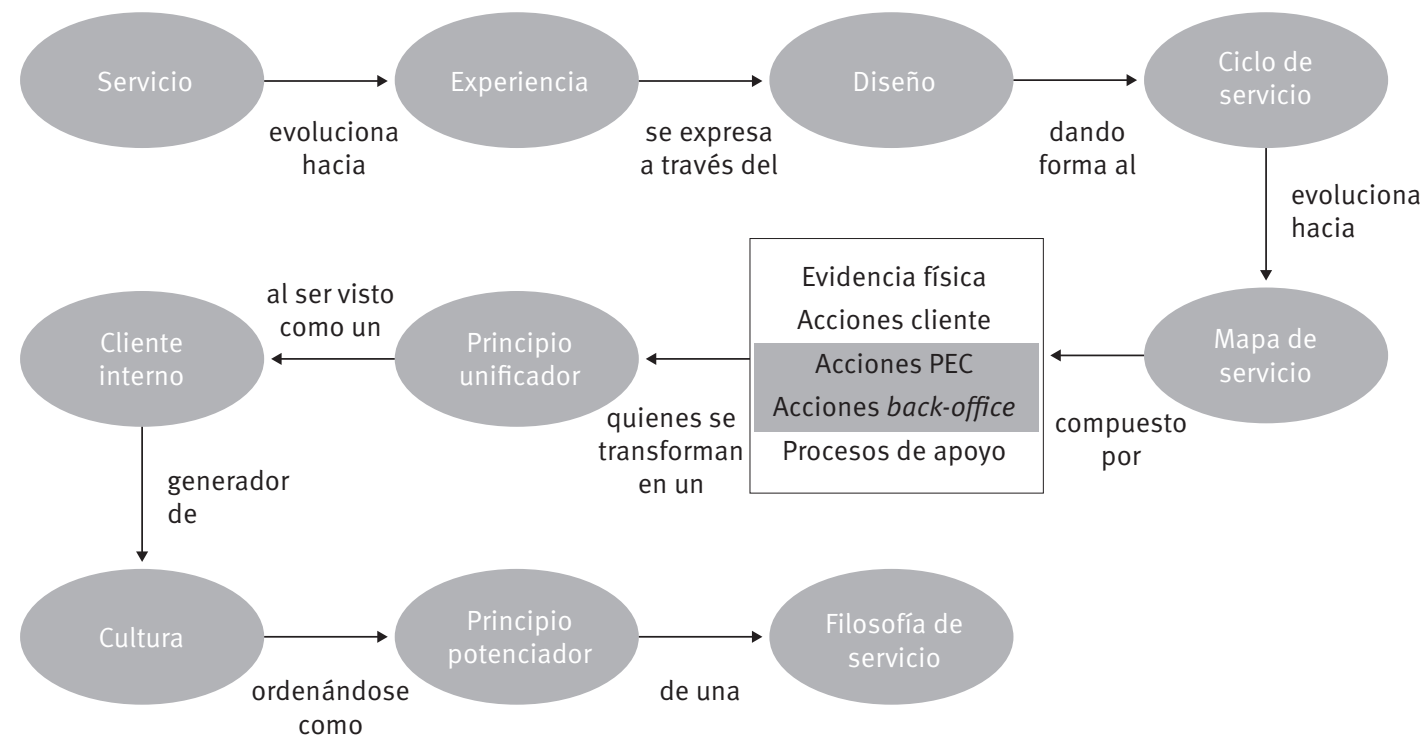


superioridad competitiva. Y como las organizaciones industriales compiten cada vez más en el área del servicio, existirá cada vez menos diferencia entre el sector industrial y el de servicios.

Parece claro que sea el "servicio" lo que esté determinando la nueva visión sobre lo que es y lo que busca -en el fondo- una organización humana. Pero la profundización en este terreno nos puede llevar a más. Si todas o casi todas las organizaciones ofrecen un servicio a la sociedad, habría que estudiar cuál es la naturaleza y contenido del "servicio", cuáles son sus características, cuáles sus prioridades, cuál su relación con las personas que conforman la sociedad y cuál su relación con las personas que integran cada organización.

\section{Naturaleza del servicio}

Mattsson (1994) afirma que los estudios muestran que los servicios son procesos en los que continuamente los clientes están involucrados como "co-productores". Por tanto, el cliente forma parte del sistema que produce el servicio.

Juran (1990, p. 15) sostiene que "servicio es el trabajo realizado para otra persona [...] El servicio también incluye el trabajo que se realiza para otra persona dentro de la empresa [...]".

Zimmermann \& Enell (1993, p. 33.2) expresan que "un servicio es un trabajo realizado para otros. [...] Los 'servicios' existen porque con ellos se pueden satisfacer unas determinadas necesida$\operatorname{des}[\ldots]$..."

Shaw (1991, p. 9) opina que "resulta más exacto y eficaz considerar el servicio como algo que cambia de modo fundamental la condición o la posición de quien lo consume".

Graterón (1995) señala que servicio es la actividad realizada en favor de otra persona. Servicio es aprovechar, valer, ser de uso o utilidad. Hacer una cosa en favor, beneficio o utilidad de otro.

Con base en estos conceptos, se puede decir que en el acto de "servir" que ofrece la organización: a) lo que se ofrece es bueno o provechoso; b) lo que se ofrece puede ser una acción, una actividad, un desempeño, un trabajo, un producto; c) lo que se ofrece es siempre para otro, no para sí mismo, sino para otra persona; d) lo que se ofrece responde a una necesidad de la otra persona; e) lo que se ofrece involucra necesariamente a la otra persona haciéndola parte del mismo servicio, y f) lo que se ofrece a esa otra persona puede producir en ella una especie de cambio.

Sale a relucir en estas afirmaciones la existencia real de una relación entre los dos polos, entre quien da el servicio y quien lo recibe, entre un "yo" que tiene la capacidad de dar un servicio determinado, y un "otro" que necesita de él. Esta relación, podemos decir, incluye incondicionalmente a "personas" que se relacionan. En este sentido, observa Lescano (2012, p. 41): “El servicio no solo tiene que ver con el desempeño de una tarea, sino que influye también en el modo de tratar y relacionarse con los demás, que depende del modo de ser de la persona. No es posible separar el servicio de quien lo brinda, pues la persona, al proporcionar un servicio, manifiesta distintos rasgos de su carácter y su estilo".

Carlzon (1991, p. 15) afirma que "cada vez más incapaces de competir desde una ventaja de orientación al producto, las economías occidentales están siendo transformadas en economías de servicio. Estamos en una encrucijada histórica donde la era de la orientación hacia el cliente ha llegado incluso para las empresas que nunca anteriormente se habían considerado como empresas de servicio".

Y así mismo, se ha reconocido también dentro de este ámbito de las relaciones personales que "como quiera que el servicio se consume en el momento que se produce, la persona que presta el servicio (ya sea banquero de inversiones o un empleado de Telefónica) ejerce una influencia superior sobre su calidad que la que conseguiría el más refinado control de calidad" (Shaw, 1991, p. 9).

Normann (1989, p. 25) apoya la misma idea cuando dice que "la mayoría de los servicios son el resultado de actos sociales que se realizan en contacto directo con el cliente y los representantes de la empresa a la que acude".

Por último, cabe destacar que si, por las anteriores opiniones, el servicio se concreta en un acto específico, pueden existir algunas características que ayuden a conocerlo y comprenderlo mejor.

Zeithaml, Bitner \& Gremler (2009) aportan cuatro características propias del servicio, a saber:

Intangibilidad: debido a que los servicios son ejecuciones o acciones en lugar de objetos, no pueden verse, sentirse, degustarse o tocarse de la misma manera que pueden sentirse los bienes tangibles.

Heterogeneidad: debido a que los servicios son ejecuciones, con frecuencia producidas por humanos, no hay dos servicios que sean precisamente 
iguales. Los empleados que entregan el servicio y los clientes pueden diferir en su desempeño.

Simultaneidad: la mayor parte de los servicios son vendidos primero y luego producidos y consumidos de manera simultánea en el tiempo, no así los productos que son producidos en un momento diferente al que son consumidos (previamente).

Caducidad: se refiere a que los servicios no pueden ser guardados, almacenados, revendidos o devueltos. No se puede contar con un "stock" o inventario de servicio. El servicio existe en el momento en que lo está recibiendo el cliente.

Rosander (1992) aporta las siguientes características, que profundizan las anteriormente nombradas:

a) Salvo algunas excepciones, los servicios no pueden medirse de la misma forma que las propiedades de los objetos o productos físicos. Esto se debe a que un servicio es algo dinámico, no estático.

b) Los servicios no pueden almacenarse. Una vez que se ha prestado un servicio, ya no existe. Puede repetirse pero no recobrarse. El servicio es un proceso, no un producto. Lo constituyen una serie de actividades afines, normalmente dominadas por la conducta humana.

c) Los servicios no pueden inspeccionarse. Los servicios pueden ser observados y sacarse conclusiones de ellos, pero no pueden pasar una inspección en el sentido literal de la expresión.

d) La calidad no puede determinarse de antemano. Los servicios no pueden examinarse de la misma manera que uno examina los alimentos antes de hacer una compra en el supermercado. El cliente no sabe si el servicio será o no satisfactorio si no lo compra y pasa por la experiencia.

e) Un servicio no tiene una vida. Los productos tienen vida. Pueden ser reparados y mantenidos. Algunos productos fallan, de manera que se puede efectuar un "test" de fallo. Los servicios no son así. Tienen duración, pero no vida.

f) Los servicios tienen una dimensión temporal. Los servicios ocurren en el tiempo. Un servicio puede consistir en una serie de actividades relacionadas en el tiempo, cada uno de cuyos componentes está sujeto al cumplimiento de la calidad. g) Los servicios se prestan tras una solicitud. Los servicios se prestan de acuerdo con dos tipos de demanda: demandas instantáneas y demandas programadas. Los primeros deben cubrir un estándar del $100 \%$ de disponibilidad y rentabilidad; mientras que los segundos responden solo a tiempos programados.

h) Los servicios son más críticos en unas industrias que en otras. Se destacan los servicios públicos y aquellos prestados en compañías en los que el error de un empleado puede no solo ser peligroso, sino fatal. En estos casos el error "cero" como meta es un deber.

i) Los servicios implican, más que fiabilidad en los productos, fiabilidad humana. La experiencia muestra la necesidad de prestar más atención a la fiabilidad en las personas. La fiabilidad humana es el complemento del error humano; así el problema se reduce a controlar, corregir y prevenir el error humano.

j) Son los empleados peor pagados en la compañía los que prestan el servicio. Esto es literalmente cierto. La calidad del servicio, en parte, viene determinada por el individuo que espera al cliente. Esto significa que la calidad de los servicios está determinada por las cualificaciones, las actitudes y comportamiento de un solo individuo o series de individuos. Así, la calidad de los servicios es muy diferente de la calidad de los productos.

k) La calidad es tanto objetiva como subjetiva. Es objetiva porque tiene que ver con las medidas, con lo observable, con hechos más allá de los sentimientos personales, con objetos materiales externos, con objetos y eventos sobre los que dos observadores independientes pudieran coincidir, con apreciaciones sensoriales. Es subjetiva, porque la afectan las apreciaciones de la mente, las reacciones del sistema nervioso, de lo que se origina de entre ellas.

En síntesis, se puede afirmar, siguiendo a Lovelock, Reynoso, D'Andrea, Huete \& Wirtz (2011, p. 4), que el servicio "es un acto o desempeño que ofrece una parte a otra. Aunque el proceso puede estar vinculado a un producto físico, el desempeño es en esencia intangible y, por lo general, no da como resultado la propiedad de ninguno de los factores de producción".

Esa búsqueda de la "calidad del servicio" ha permitido entender que lo importante es 
desarrollar la "capacidad de servir" como objeto de la tecnología y el conocimiento.

\section{Un salto evolutivo hacia la experiencia del servicio}

Para comenzar a profundizar acerca de la importancia del servicio en las organizaciones, lo primero es poner en contexto la evolución del servicio. Pine \& Gilmore (1998) establecen un modelo de evolución del valor económico, que comienza en una posición competitiva de indiferenciación con la extracción de commodities, avanza en la creación de bienes, llegando a un punto de mayor diferenciación a través de la entrega de servicios. Pero existe un punto más alto de diferenciación competitiva: la puesta en escena de experiencias. Así, Pine \& Gilmore (1998, p. 98) plantean que:

Una experiencia ocurre cuando una compañía utiliza servicios como el escenario, y los bienes como utilería, para comprometer a los consumidores individualmente de manera que crear eventos memorables. Los commodities son fungibles, los bienes tangibles, los servicios intangibles, y las experiencias memorables [ver figura 2].

Otra definición de Pullman \& Gross (citados en Zomerdijk \& Voss, 2010) es que una experiencia se produce cuando un cliente tiene alguna sensación o adquiere conocimiento a partir de una interacción con elementos de un contexto creado por un proveedor de servicio.
Morrás \& Polizzi (2002, p. 5) definen experiencias como: "procesos generativos (autorreforzantes) productores de efectos corporales, emocionales o cognitivos que ocurren en las personas como respuesta a un conjunto de estímulos percibidos de naturaleza física, mental o virtual".

El correcto diseño del servicio en el encuentro con sus clientes se transforma, por tanto, en un eje fundamental para alcanzar el compromiso y fidelización de los clientes. Zomerdijk \& Voss (2010) establecen que el compromiso puede ser de carácter emocional, físico, intelectual o espiritual, y que se pueden alcanzar distintos niveles de compromiso de acuerdo con el nivel de colaboración de los clientes y de la conexión con el ambiente. Estos autores también plantean seis proposiciones acerca de las organizaciones de servicio centradas en la experiencia de sus clientes, que establecen que el diseño de servicios centrados en la experiencia involucran: a) el diseño de una serie de encuentros de servicios y señales; b) diseño sensorial; c) empleados de primera línea comprometidos con sus clientes; d) prestar atención a la estructura dramática de los eventos; e) prestar atención a la relación entre los clientes durante la entrega del servicio, ya que otros clientes van a participar en la vivencia de la experiencia, y f) acoplar estrechamente a los empleados back office con la experiencia que va a vivir el cliente.

A partir de lo anterior, se puede decir que las experiencias son creadas por un proveedor de servicios, inducidas externamente, y que para lograr que estas sean memorables y únicas para

FIGURA 2. La progresión del valor económico

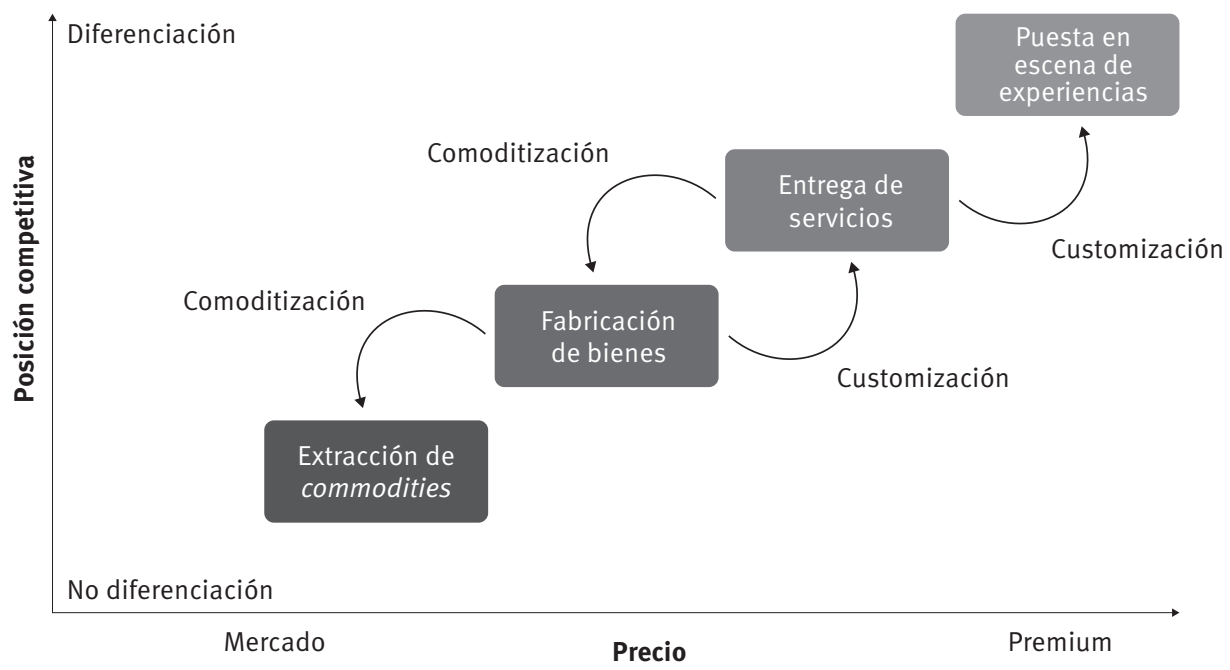


el cliente, que generen valor y sean relevantes, el diseño de esas experiencias desde dentro de la organización es fundamental. A pesar de que la vivencia es personal, la organización puede crear acciones que moldeen esas experiencias de algún modo y lograr buenos resultados.

\section{El cliente y los "momentos de la verdad"}

A la persona objeto del "servicio" específico que presta una organización humana se le suele llamar "cliente". Es decir, que el servicio no existe sin este último. Y se hacía antes mención de dos polos en la relación del "servicio", de dos personas. Pues bien, uno de los polos tiene determinadas necesidades insatisfechas, las cuales manifiesta a través de una demanda de productos o servicios. Este polo es el principio y fin de todo proceso. Por medio de su demanda se inicia el proceso, y al satisfacer dicha demanda queda concluido el proceso. Se hace referencia a él como "cliente".

Horovitz (1990, pp. 2-3) opina que "el servicio es el conjunto de prestaciones que el cliente espera, además del producto o del servicio básico, como consecuencia del precio, la imagen y la reputación del mismo". Se puede entender así que cada relación en el momento en que se produce el "servicio" es un contacto integrado por una serie de pasos o procesos, los cuales determinan el éxito del negocio o la satisfacción total del mismo. Tal satisfacción dependerá de una serie de cualidades que solo los seres humanos podemos medir y, por supuesto, necesitar. Para Senlle (1993, p. 89), "la palabra cliente define un grado de fidelidad, simpatía, agrado y satisfacción. Nos sentimos clientes cuando se satisfacen nuestras necesidades y somos bien tratados".

Esto se puede concretar aún más si se estudia el "contacto" o "contactos sucesivos" entre la organización y el cliente: cada vez que el cliente interactúa con la empresa ocurre algo. Ocurre lo que los especialistas han denominado "momento de la verdad".

Albrecht (1992, pp. 30-31) define y explica los "momentos de la verdad":

El momento de la verdad -expone- es cualquier situación en la que el cliente se pone en contacto con cualquier aspecto de la organización y obtiene una impresión sobre la calidad de su servicio. La expresión española 'momento de la verdad', que tiene su origen en las corridas de toros, señala el momento final en el cual se enfrentan, solos, el matador y el toro. [...] Por supuesto, quisiéramos emplear el término sin sus alusiones a la confrontación, para sugerir que el cliente y la persona que le presta el servicio se pone en contacto en muchos episodios críticos, y que el cliente se forma su impresión acerca de la calidad del servicio en dichos encuentros. De modo colectivo, todos ellos conforman la imagen general del servicio de la empresa.

Al resumir las anteriores aportaciones se tiene que el cliente es quien necesita del "servicio" específico de la organización. En la relación "organización-cliente" hay una serie de contactos que influye en la percepción del cliente respecto de la empresa y que concluye con una satisfacción o insatisfacción del negocio, transacción o beneficio adquirido. La satisfacción del cliente dependerá del grado de cualidades objetivas y subjetivas que tiene el contacto con la organización. Generalmente tiene una influencia positiva la atención esmerada y el trato cordial, lo que puede multiplicar la buena imagen de la empresa. Al contrario, el trato descortés y poco amable tiende a ser suficiente para opinar desfavorablemente de esta.

Cada contacto del cliente con cualquiera de las partes de una organización suele llamarse "momento de verdad" o "momento de la verdad". Son muchos los episodios críticos que permiten palpar la "calidad del servicio", y, en resumen, de modo colectivo, la imagen de la empresa. Según los autores, el conocimiento y uso del concepto "momento de la verdad" por parte de los empleados y gerentes ayudaría a tener una visión más concreta sobre la satisfacción del cliente.

La unión de cada uno de estos momentos de la verdad vividos por el cliente conforman el ciclo de servicio.

Una idea introducida por Albrecht (1992) es el "ciclo del servicio", que consiste en darse cuenta de que los momentos de la verdad no son únicos sino que se producen en grupos o conjuntos (ver figura 3).

Lo que en realidad ocurre es que el cliente decide hacer negocios con una organización, y atraviesa una serie de momentos de la verdad, los cuales forman parte de una experiencia relativa a la totalidad del servicio o del ciclo de servicio.

El concepto de ciclo de servicio ayuda a las personas a ofrecer asistencia a los clientes, permitiéndoles reorganizar las imágenes mentales de lo que ocurre. 
FIGURA 3. El ciclo del servicio según Albrecht

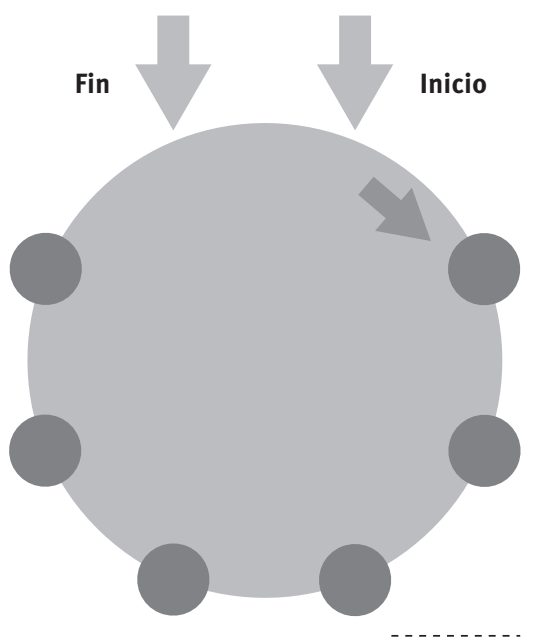

Fuente: adaptado de Albrecht, 1992

La construcción básica del servicio ya no es tarea del empleado, sino que ahora se convierte en el momento de la verdad que él mismo controla. El empleado ya no fabrica el producto, sino que es parte del producto. La calidad ya no es una ejecución satisfactoria de la tarea asignada, sino que ahora se la define como "el momento de la verdad".

Esta idea del "ciclo del servicio" enlaza con la reconocida opinión que destaca la importancia de interesarse por la "experiencia entera" de relación entre el cliente y el producto.

Graterón (1995) también habla del ciclo del servicio, pero destaca que este es una secuencia repetible de acontecimientos en que diferentes personas tratan de satisfacer las necesidades y expectativas del cliente en cada punto de un ciclo que empieza con el primer contacto entre el cliente y la organización, y termina cuando el cliente considera que el servicio está completo, para reiniciarse cuando el cliente decida regresar por más (ver figura 4).

Conviene destacar que cuando el cliente hace el primer contacto con la organización, no aprecia solo uno de los elementos, sino que, con base en su propia experiencia y percepción, aprecia un panorama completo de la organización; es decir, visualiza "el ciclo del servicio".

En síntesis, el "ciclo del servicio" (figuras 3 y 4), según las anteriores aportaciones, son el conjunto de "momentos de la verdad" por los que un cliente atraviesa al relacionarse con la organización. Es una experiencia de sucesivos momentos
FIGURA 4. El ciclo del servicio según Graterón

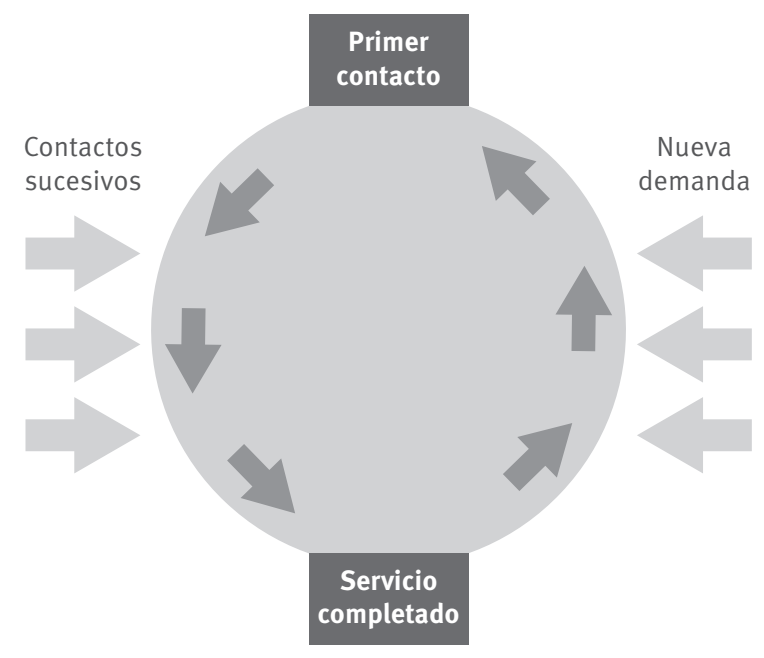

Fuente: adaptado de Graterón, 1995

que al final permiten hacerse una visión total del "servicio". Se usa como medio para ayudar al empleado a modificar su punto de vista parcial sobre la organización en la que forma parte. Así, podrá considerar a los clientes como los clientes lo consideran a él. Una de sus consecuencias más inmediatas -como ciclo de experiencia- es el renovado deseo del cliente en volver a la organización por mayor demanda.

\section{La evolución del ciclo de servicio: el mapa de servicio}

En la actualidad, se puede observar que con el tiempo algunas herramientas de diseño de servicios han evolucionado en su especificidad, como es el caso del ciclo de servicio.

Una herramienta de diseño más completa que incluye el ciclo de servicio sería el "mapa de servicio" o Service Blueprint, útiles en la contextualización y el desarrollo de la experiencia del cliente. El desarrollo de un mapeo de servicios requiere identificar todos los momentos de la verdad, y la especificación de las relaciones entre los participantes que actúan en cada uno de ellos (Lovelock et al., 2011).

Zeithaml et al. (2009) explican que en un mapa de servicio, como el que se presenta en la figura 5, existen acciones del cliente, lo cual abarca los pasos, elecciones, actividades e interacciones que realiza el cliente en el proceso de compra, consumo y evaluación del servicio. Pero en paralelo a 
FIGURA 5. Mapa de servicio
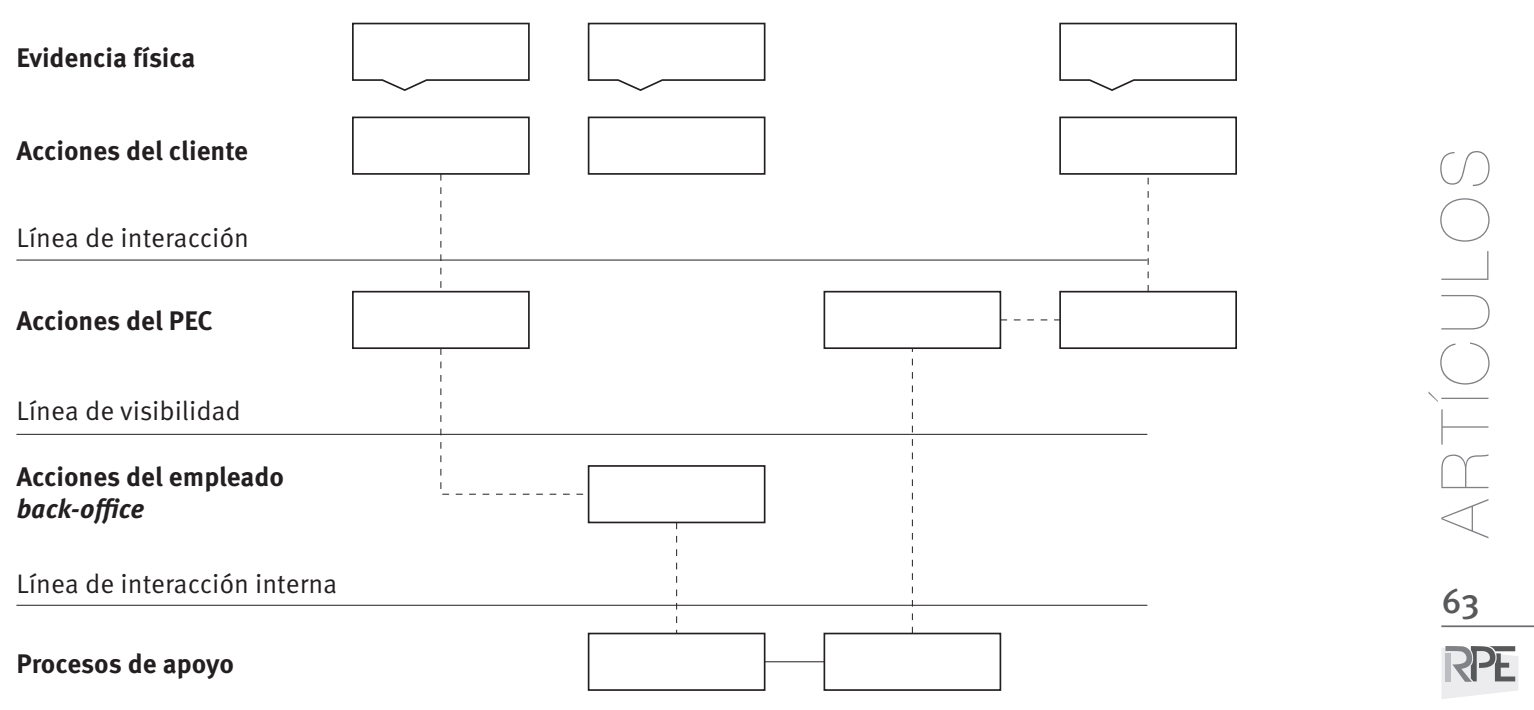

Fuente: adaptado de Zeithaml, Bitner y Gremler, 2009

las acciones del cliente hay dos áreas de acciones de los empleados. Una es totalmente visible para el cliente y se denomina acciones del personal en contacto (y que en la figura 5 aparece con las siglas PEC). La siguiente no es visible para el cliente y se designa como acciones del empleado back office. Por último, están los procesos de apoyo necesarios para poder generar dichas interacciones. Adicionalmente, se identifica qué elementos de la evidencia física son los relevantes en cada uno de estos momentos de interacción. Las cuatro áreas de acción clave están separadas por tres líneas horizontales. La primera es la línea de interacción, que representa las interacciones directas entre el cliente y la organización. La siguiente es la línea de visibilidad, que simboliza la separación de todas las actividades de servicio visibles para el cliente de aquellas que no lo son. Esta línea también separa lo que hacen los empleados en contacto (PEC) de lo que hacen entre bastidores. Y la tercera es la línea de interacción interna, la cual separa las actividades del empleado back office de aquellas otras áreas internas que apoyan los procesos de servicio.

El mapeo se utiliza como herramienta de apoyo que traza visiblemente parte de la operación del servicio, de cara al cliente. A partir de la declaración de los momentos de la verdad, los actores involucrados en cada uno, y los procedimientos necesarios que debiesen ocurrir, la organización puede dilucidar de una manera más clara sus puntos críticos de contacto y las fallas que pudiese estar cometiendo. El mapeo, por tanto, lleva a contribuir en el diseño y en la implementación de la experiencia memorable que se busca para generar valor y fidelización al cliente, ya que centra la atención en lo importante para los clientes en cada interacción con el servicio.

Y es aquí donde se vislumbra el protagonismo de quienes tienen como rol fundamental el propiciar la implementación y entrega de servicios. Todo esto, de una manera alineada y coherente, que le permita al cliente concretar sus acciones de manera exitosa. Es necesario enfatizar que el accionar del personal en contacto y del back office, entre otros aspectos, es lo que determina, en gran medida, la existencia de una experiencia memorable para el cliente.

Es a ellos a quienes se les otorga la labor de "servir" durante el proceso, y no solo de servir al cliente, sino de lograr que la entrega de servicios se funde desde los procesos más internos de la organización, y, de esta manera, se produzca una perfecta reacción en cadena, en donde la colaboración entre personas es la clave, y la experiencia exitosa, el resultado.

\section{El servicio como principio unificador de la organización}

Han servido las anteriores explicaciones y descripciones para visualizar la importancia de 
este novedoso concepto en el que "servir" y "capacidad de servir" conforman el principio unificador de la organización. Carlzon (1991, p. 15) lo confirma exponiendo que:

En el mundo de hoy, el punto de partida debe ser el cliente -no el producto o la tecnología en sí mismos-y esto significa que las compañías deben organizarse de forma diferente para sobrevivir. En una compañía orientada hacia el cliente, la distribución de papeles es radicalmente diferente. La organización se descentraliza, delegando responsabilidad a aquellos que hasta ahora habían formado parte del nivel más bajo de la pirámide y que obedecían las órdenes. La estructura tradicional de la corporación jerárquica está comenzando a ceder a una estructura más horizontal. Esto es particularmente verdad en las empresas de servicios que empiezan no por el producto sino por el cliente.

Como se advierte, al pensar en el servicio que hay que prestar a los clientes, toda la organización se posesiona de una idea que la transforma de tal modo que la estructura de la misma no es solo consecuencia de un "organigrama" preestablecido ni de un número de funciones o tareas, o resultados específicos esperados. La transformación de la organización surge de la reorientación de sus fuerzas hacia el "servir al cliente" como centro de contactos o relaciones necesitadas de calidad para mantener "viva" a la empresa en el ámbito del mercado, de la competencia y de la demanda.

La reorientación de la organización hacia el "servir al cliente" se convierte en un valor que "une". Y "unir" significa juntar, enlazar, ligar, atar, fundir, mezclar, ensamblar, incorporar: palabras o ideas necesarias para una organización integrada por personas que, aunque tienen cada una intenciones (particulares) distintas, se "re-unen" (o así lo intentan) para satisfacer necesidades personales y sociales; necesidades muy relacionadas con actividades y procesos que no tienen otro fin que el de hacer o dar un bien a otro, un servicio a la persona (cliente) y a la sociedad.

El servicio "une" porque, como expresan Thornely \& Lees (1993), es "una causa noble", es decir, una de las más poderosas motivaciones. Hacer ver con claridad que la gente puede servir a otros, ayuda a entender que trabajan juntos por "una causa noble".

Otra consecuencia de este estar unidos alrededor del "servir al cliente" es la de reconocer que los miembros de la organización se hacen un bien a sí mismos y un bien a la sociedad cuando ven con claridad que "sirven" a otros con su trabajo individual y grupal. Y porque, sin duda alguna, servir es propio de ser "persona" en el hecho de "darse", de "estar al servicio de los demás".

En efecto, Frankl (1984, pp. 26-27) denomina "autotrascendencia" a esa capacidad de darse que tiene el hombre, la cual:

Denota el hecho de que el ser humano siempre apunta y se dirige a algo o alguien distinto de sí mismo - para realizar un sentido o para lograr un encuentro amoroso en su relación con otros seres humanos-. Solo en la medida en que vivimos expansivamente nuestra autotrascendencia, nos convertimos realmente en seres humanos y nos realizamos a nosotros mismos. Esto siempre me hace recordar el hecho de la capacidad del ojo de percibir visualmente el mundo que le rodea, la que irónicamente es contingente de su incapacidad para percibirse a sí mismo. Cada vez que el ojo ve algo de sí mismo, su función está perturbada. Si yo estoy afectado por una catarata, veo una nube -mi ojo ve su propia catarata-. 0 si estoy afectado por un glaucoma, veo un halo como el arco iris alrededor de las luces; es como si mi ojo percibiera la tensión ocular aumentada producida por el glaucoma. El ojo que funciona normalmente no se ve a sí mismo, no se percibe a sí mismo. Análogamente, nosotros somos humanos en la medida en que somos capaces de no vernos, de no notarnos y de olvidarnos de nosotros mismos dándonos a una causa para servir, o a otra persona para amar. Sumergiéndonos en el trabajo o en el amor, nos estamos trascendiendo, y, por tanto, nos estamos realizando a nosotros mismos.

Pero esta idea de centrarse en el cliente se complementa aún más, cuando no solo se mira hacia afuera de la organización, sino cuando la propia organización se mira hacia adentro. Allí, en esa búsqueda de la "sinergia interna", encuentra la organización humana el principio vital de su propio desarrollo y el de cada uno de sus miembros. Y desde allí, incluso más, el atender con calidad al cliente sería una de las consecuencias de esa revolución interior.

\section{Servicio al cliente interno}

Se ha visto con claridad cómo una organización centrada en el "cliente externo" puede lograr una mejoría inusual. Se trata, en síntesis, de tener una visión concreta del concepto "servicio", 
su naturaleza, el concepto de "cliente", de "experiencia", de "momento de la verdad", el "mapa de servicio" y la consecuente conclusión por la cual se ha determinado que pensar y poner en práctica estos conceptos e ideas ayuda en mucho a la unidad de la organización, y a la calidad personal de sus miembros.

Pero volviendo sobre el concepto que expuso Juran (1990) acerca del "servicio", él lo complementa con la siguiente frase: "El servicio también incluye el trabajo que se realiza para otra persona dentro de la empresa [...]". La palabra resaltada en cursivas es del propio autor, por la que nos hace entender el interés que tiene para el mejoramiento vital de la calidad mirar e interesarse en los aspectos internos de la misma, y en concreto, en el recurso humano: la persona "dentro".

Albrecht (1992, p. 26) expone que:

Lo que debemos buscar en la gestión y en el funcionamiento de empresas que prestan servicios excelentes no es más que una sinergia interna completa. Esto se logra centrando las energías en comprender qué significa, globalmente, el negocio, el ordenamiento de recursos que está por detrás del envío del producto en los momentos de la verdad y el trabajo en equipo, posibilitado por el hecho de que cada grupo comprenda cuál es su aportación al éxito de la empresa.

Eso de comprender globalmente a la organización -como señala Albrech- es lo que se busca al estudiar a los miembros que laboran dentro de ella, es decir, es lo que en cierta forma permite entender cómo se logra "el servicio al cliente interno". Por ello es necesario definir primero al "cliente interno" con claridad, y en los términos en que los distintos autores lo hacen.

Juran (1990, p. 16) explica que "los clientes internos son afectados por el producto y también son miembros de la empresa que los produce. A menudo se les llama clientes, a pesar del hecho de que no lo son en el sentido que da el diccionario; esto es, no son compradores".

Luego Albrecht (1992, p. 26) en sus conferencias a directivos para hacerlos reflexionar bromea diciéndoles:
Si no saben quiénes son sus clientes, pueden hacer una prueba muy sencilla para descubrirlo. Solo dejen de hacer lo que hacen por espacio de dos semanas y vean quiénes son los que se empiezan a quejar. Esos son sus clientes. [...]. En realidad, este no es un mal modo de hacerlo, al menos mentalmente. Comience por preguntarse: “ $¿ A$ quién le produciría un inconveniente, y por qué, si mi departamento dejara de funcionar?" "¿Qué departamentos o personas tendrían dificultades para cumplir sus misiones sin los resultados que les ofrecemos nosotros?” “QQuién se vería afectado directamente y quién indirectamente?” Empiece a tomar notas de sus respuestas.

Según lo anterior, en resumen, el "cliente interno" es la persona más inmediata a nosotros dentro de la organización. Es la persona más afectada por la calidad de nuestro trabajo. Y dicha calidad también puede afectar indirectamente a otros clientes internos que siguen en la cadena, bien sea a una persona o a un conjunto de las mismas.

Existen, por su parte, otros autores que sin contradecir lo ya mencionado, más bien lo explican desde otra perspectiva. Se trata, por ejemplo, de pensar en la organización como sistema de procesos. Y es que, como dice Scholtes (1991), los conceptos de consumidores y proveedores se deducen fácilmente una vez que se comprenda la idea de un proceso: las personas u organizaciones que proceden a la serie de actividades que identificaron como proceso son "los proveedores", y aquellos que siguen, que usan el producto o servicio, son los "consumidores".

Con el mismo sentido, pero de otra manera, lo expresa Senlle (1993) en la figura 6.

Cada trabajador compra (C), transforma (T) el trabajo y vende (V) al compañero siguiente. Si en esta cadena una persona hace el trabajo descuidadamente, con errores o fallos, esto exige al comprador dedicar un tiempo extra a revisar y arreglar trabajos que eran de la responsabilidad del vendedor.

La calidad del servicio de cualquier organización humana está dependiendo constantemente

FIGURA 6. Comprar, transformar y vender

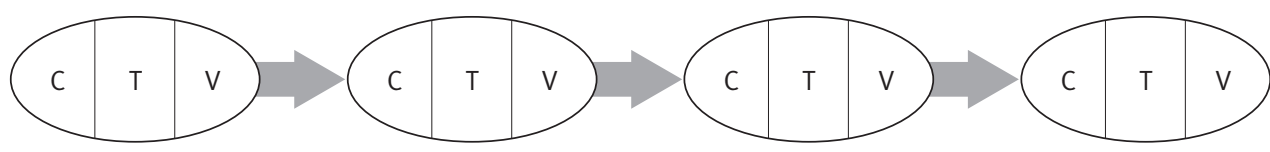

Fuente: adaptado de Senlle, 1993 
de la forma como unos entregan a otros la parte (procesos) que les ha tocado mejorar o atender.

Todo ello rebasa la estricta línea vertical, afirman Ginebra \& Arana de la Garza (1991, p. 99), y conduce a la necesidad [...] de ocuparse de la línea horizontal de la calidad. Una y otra línea se funde en un conjunto indivisible. No podemos departamentalizar la calidad. Todos en la empresa son productos, todos en la empresa son cliente, todos en la empresa son proveedor; todos en la empresa son base de calidad y servicio.

En forma de resumen, y como síntesis de lo expuesto, Heskett, Jones, Loveman, Sasser \& Schlesinger (1994) aportan que ellos llaman internal quality (calidad interna) al ambiente de trabajo que contribuye mejor a la satisfacción de los empleados, entendiendo por ello los sentimientos que los empleados tienen hacia la compañía, su trabajo y colegas; y por otro lado, a las actitudes que la gente tiene hacia los demás y el modo como esa gente sirve a cada quien dentro de la organización.

Así pues, al hablar de servicio al cliente interno, se está dando paso a la profundización en el análisis de los procesos internos de la organización humana, que de alguna forma permiten, por sí mismos, desarrollar la calidad absoluta de todo lo que integra y, en especial, de las personas que la hacen posible día a día.

A continuación se presenta un conjunto de ideas complementarias a los anteriores planteamientos, las cuales sirven para orientar, de manera más concreta, a la organización hacia adentro, hacia sus "clientes internos", o en otras palabras, hacia el principio unitivo ya comentado, y ahora muy especialmente, hacia el principio potenciador.

\section{Cultura del servicio}

Diversidad de autores hablan en la actualidad de ese básico o fundamental contexto que permite motivar, dirigir y orientar las conductas, las actuaciones, los logros en las organizaciones humanas. Parece ser que el proceso de conformación de una cultura, dentro de una organización, sea lo que esté llevando a resultados positivos cuando se trata de buscar mejoras, nuevos resultados, nuevas direcciones, nuevas orientaciones.

Por ejemplo, Albrecht (1992, p. 26), expresa que:
Es un enorme desafío lograr que el personal interno de una organización de servicio piense, actúe y hable sobre cuál es su participación en el éxito del negocio desde la perspectiva del cliente. Nunca se logra que alguien esté completamente alerta, pero en las organizaciones que prestan excelentes servicios, la mayor parte del personal interno ha aprendido a incluir tareas en el contexto del éxito de la compañía dentro de su mercado.

Con esto se puede afirmar que para impregnar a una organización de los conceptos anteriores de "servicio", "calidad del servicio", "momentos de la verdad", "servicio al cliente interno", etc., se necesita aparentemente la actualización de una cultura, o la incorporación de ideas y modos de hacer en una cultura, lo que convertiría a la cultura de esa organización en una "cultura de servicio" o en una "nueva cultura".

Sin embargo, ¿qué es una cultura dentro de la organización y qué importancia puede tener una cultura de servicio en una organización?

Lassey \& Marshall (1988, p. 243) dicen que la cultura "consiste en una larga serie de presunciones asumidas e implícitas que abarcan la visión que los miembros del grupo tienen tanto sobre sus relaciones externas en sus distintos entornos, como sobre sus relaciones internas con los demás".

Por cultura, Leal \& Valle (1989) entienden aquel conjunto de creencias y valores compartidos, que da forma al significado que de la organización tienen sus miembros y que les suministran las reglas de conducta, estilos de comportamiento y un alcance de la realidad y de su entorno circundante.

Hellriegel \& Slocum (2009, p. 458) expresan que la cultura organizacional "refleja los valores, creencias y actitudes que han aprendido y que comparten sus miembros".

Los autores Robbins \& Judge (2013, p. 512) conciben la cultura organizacional como un "sistema de significados compartido por los miembros, el cual distingue a una organización de las demás".

Lo que guía el estilo de las relaciones entre los miembros o el conjunto de creencias que se comparten -o el conjunto de valores que comparten, o lo que da sentido al trabajo, o lo que estimula a superarse- pareciera ser lo que define una cultura organizacional.

Según estas anteriores aportaciones, la cultura determina los puntos de referencia que motivan 
y orientan las actuaciones. Por tanto, es lógico plantearse que sea el valor "servicio" un claro punto de referencia, un valor cultural, o un valor que crea una cultura dentro de una organización.

Pero lo primordial aquí y ahora consiste en el cómo crear esa cultura del servicio. Deal y Kennedy, citados por Albrecht (1992, p. 115), dicen más específicamente que "una cultura de servicio es una forma de hacer las cosas que valora enormemente la calidad del servicio, puesto que esta cumple una función básica en el éxito de la empresa".

Estas contribuciones destacan la importancia de reconocer que la "cultura de servicio" es solo un elemento sobre el cual se busca empapar a la organización (con su cultura específica) en dirección al "servicio", y por tanto a la "capacidad de servir" que se destacaba en un apartado anterior.

Pero la cultura, entendida como "conjunto de creencias, normas y valores que forman la base de la conducta colaboradora, humana [...] se puede cambiar en las siguientes organizaciones empresariales, y con total efectividad si se cumplen las condiciones precisas", opina Normann (1989, p. 185).

Esto lo confirma Albrecht (1992) cuando plantea que cree totalmente en una "cultura de servicio", y destaca cuáles son sus características distintivas:

a) existe una visión o un concepto claro del servicio;

b) los ejecutivos enseñan constantemente lo que es el servicio;

c) los gerentes toman como modelo el comportamiento de que el cliente está primero;

d) se espera un servicio de calidad de todas las personas involucradas;

e) se recompensa el servicio de calidad.

Un ejemplo de lo anterior, reportado por Hellriegel \& Slocum (2009), es el caso de TDIndustries. Dicha empresa lleva más de 25 años practicando el liderazgo servidor. Se trata de una empresa del ramo de la mecánica, la electricidad y la plomería, que vale más de 250 millones de dólares. De forma continua ha quedado clasificada dentro de la lista de las 100 mejores empresas para trabajar en Estados Unidos que publica la revista Fortune. La empresa tiene más de 1500 empleados, y muchos de ellos llevan más de 10 años trabajando allí. Su presidente considera que el éxito de la empresa se debe a su misión, su cultura y su práctica del liderazgo servidor. Este "liderazgo servidor" significa que toda persona puede llegar a ser líder si primero sirve a otros, y después, por propia decisión, lidera. Esto significa que los líderes (servidores) son coach de sus empleados (líderes) sirviéndoles para que satisfagan sus necesidades. Por ejemplo, el presidente de TDIndustries, en su rol de servidor, contesta su teléfono, no tiene espacio exclusivo en el estacionamiento y trabaja, como todos, en un cubículo de $3 \times 4$ metros.

\section{El triángulo del servicio interno}

Albrecht (1992) propone otra figura orientadora: el triángulo del servicio interno (figura 7), con el que representa a la exitosa organización de servicios, la cual cuenta con empleados comprometidos con el servicio, y quienes toman sus pautas de tres elementos existentes en su entorno:
a) la cultura de la organización;
b) la dirección a la que están sujetos;
c) la estructura de la organización.

El triángulo del servicio interno sugiere que el empleado de la línea operativa necesita el respaldo de las tres fuentes: la cultura, los líderes y la estructura, y los sistemas de la organización. Si todos estos elementos se encuentran perfectamente alineados detrás del empleado, este pondrá lo mejor de sí para prestar el servicio, ya sea al cliente externo o al interno.

FIGURA 7. El triángulo de servicio interno

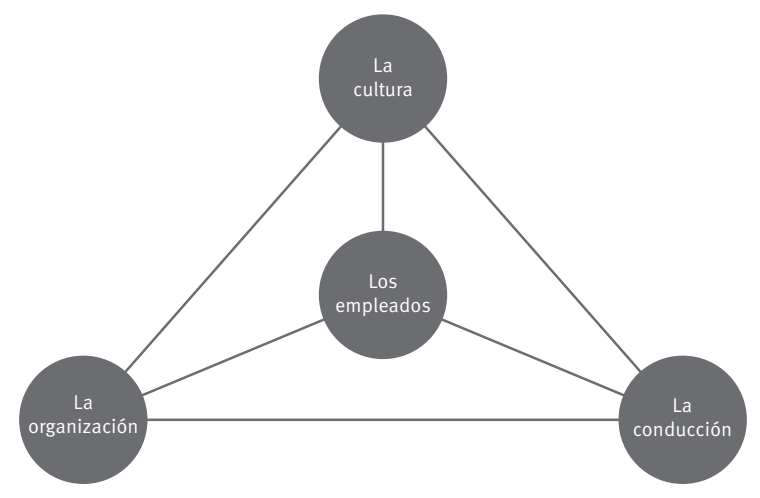

Fuente: adaptado de Albrecht, 1992 
Con esta anterior visión, en síntesis, la organización humana basa su realidad, más que en un ámbito de relaciones de poder, función o cargos, en un ámbito de relaciones de verdadero servicio a las personas con quienes se labora. Incluso cuanto más alto se está en un cargo, más se sirve porque más clientes internos se tienen.

\section{El servicio al cliente interno como principio potenciador de la organización}

Ginebra \& Arana (1991) plantean que es necesario analizar el diario hacer de los empleados preguntándoles: Todo esto que haces, ¿para quién lo haces?, ¿quién lo compra? Estas preguntas dan una dimensión diferente al hacer: le confieren la finalidad de "servir para". Es lo único que vincula el hacer diario al servicio: mi labor, mi puesto tiene sentido en la medida en que sirvo a través de este y me vinculo con las necesidades del cliente externo.

Todo lo anterior determina el cambio de las relaciones de servicio. Pero el cambio no es fácil. Primero hay que resaltar que todo puesto de trabajo se justifica por la relación de unos con otros. Así, todos somos clientes de alguien, y proveedores de otro. Esta dualidad transforma actitudes y facilita el nuevo enfoque.

Es preciso romper feudos, cambiar el sentido de la autoridad, entender que ya no es válido contentar al jefe, etc. Voltear el organigrama implica que el jefe está para servir al subordinado, para facilitarle su trabajo. Y cuanto más alto está en el organigrama es más importante, no por "mandar a muchos" sino por "servir" a muchos; esta es la premisa básica del director general.

Temple \& Droege (1994) destacan también la importancia de centrarse en la idea de satisfacer las necesidades de "cliente interno" para lograr cambios dentro de la organización, postura que confirman Schneider \& Bowen (1993) cuando exponen que no solo ha de prestarse atención a los clientes, sino también a los empleados, ya que el ambiente interno de la organización se refleja directamente en los clientes externos. Y basan esta afirmación en estudios realizados en los Almacenes Sears, en NCR, en Ryder Truck, en 30 Bancos del Oeste Medio de los Estados Unidos, y en 200 establecimientos de una gran cadena de almacenes, cuyos resultados arrojaron que existe una estrecha relación entre la satisfacción interna de los empleados y su incidencia positiva sobre los clientes externos.

Dos estudios recientes dan cuenta de que los empleados tratan a los clientes de la misma forma en que la organización los trata a ellos. Un estudio reunió datos de 292 gerentes, 830 empleados y 1772 clientes bancarios en Japón. Los investigadores examinaron si las compañías que proporcionaban prácticas laborales de alto desempeño, como capacitación de servicio, información compartida, equipos autoadministrados y trabajadores con autonomía, tenían un mejor ambiente para el servicio al cliente, y si este ambiente de servicio estaba relacionado con un mejor desempeño organizacional. Los resultados arrojaron que las prácticas que benefician a los empleados favorecieron un mejor desempeño a través de un ambiente positivo para el servicio al cliente (Liao, Toya, Lepak \& Hong, 2009).

La otra investigación estudió el "servicio" interno, es decir, el grado en que los empleados creen que sus unidades de trabajo reciben un buen trato de la organización en su conjunto. Más de 600 empleados de una empresa de servicios financieros del Caribe describieron su servicio interno, y después se reunieron datos sobre la calidad de casi 2000 clientes. Los resultados demostraron que el ambiente de servicio se relacionaba más de manera positiva con la satisfacción del cliente cuando el servicio interno era elevado; es decir, que un ambiente positivo para el servicio produce clientes más satisfechos, sobre todo cuando la organización brinda un servicio interno positivo a sus empleados (Ehrhart, Witt, Scheider \& Perry, 2011).

Estas ideas y estos resultados llevan a pensar que la organización humana orientada hacia el servicio al cliente interno tiene una mayor posibilidad de potenciar sus innovaciones, y de desarrollar, por tanto, sus proyectos, planes y estrategias.

Potenciar significa, según la última edición del Diccionario de la Real Academia de la Lengua Española (2014), "comunicar potencia a una cosa o incrementar la que ya tiene". Mediante el servicio y la capacidad de servir al cliente interno, lo que se busca es eso: incrementar las posibilidades que la misma organización ya tiene: hacer posible, facilitar y fomentar la capacidad de servir de la misma; una capacidad, por cierto, muy propia de la persona humana, y que tiene que ver con esa inmensa necesidad que tiene de ser amistosa.

Toda organización humana basada en el servicio al cliente interno, incluso sin pensar en ello 
sistemáticamente, es un ámbito de relaciones. Afirma Pérez López (2014, p. 65):

Sabemos muy bien que las relaciones de tipo espontáneo entre el director de producción y el director de ventas tienen bastante importancia a la hora de asegurar una adecuada coordinación de ambas funciones en cualquier empresa. Si ambos directores son los que normalmente denominamos "muy buenos amigos", hay grandes posibilidades de que bastantes problemas en el área de ventas sean resueltos gracias a unos esfuerzos extraordinarios en el área de producción, y lo mismo cabe decir a la inversa. Todo ello vendría a significar una buena coordinación entre ambas fuentes. Si las relaciones entre ambos directivos fuesen aquellas que suelen existir entre enemigos declarados, lo más probable es que la coordinación entre producción y ventas llegase a construir un problema muy grave.

Las relaciones amistosas y cordiales entre los miembros de una organización humana se fundamentan ya desde tiempos antiguos. En la sociedad clásica de la antigua Grecia, Aristóteles se atrevía a decir que "la amistad es una virtud o va acompañada de una virtud y es, además, la cosa más necesaria en la vida. Sin amigos nadie escogería vivir, aunque tuviese todos los bienes restantes" (1973, p. 102). Lo mismo señala de la necesidad de concordia y amistad en las ciudades, teniendo en cuenta que "la más alta forma de justicia parece ser una forma amistosa". Y más adelante trata de las restantes comunidades y asociaciones que siendo parte de la "comunidad política" las une una forma de amistad (Aristóteles, 1973, p. 110).

Así, se puede decir que el servicio dentro de la organización se orienta a la búsqueda de un bien que el ser humano necesita. Un bien que en cierta forma potencia las capacidades de cada individuo, y a la institución misma como conjunto de esos individuos. Apunta esta orientación, sin duda alguna, a aquella afirmación de Juan Pablo II, citado por Melé Carné (1992, p. 26), que expone que la empresa "debe transformarse en comunidad de vida, en un lugar donde el hombre convive y se relaciona con sus semejantes; y donde el desarrollo personal no solo es permitido sino fomentado".

La organización humana orientada hacia el servicio al cliente interno encuentra su dinamismo en las relaciones de servicio entre sus miembros. Se potencia así la organización, haciéndola pasar de un ámbito de relaciones basadas en los cargos o funciones, a un ámbito más propio de la naturaleza humana: un ámbito apoyado en relaciones de servicio, en relaciones donde interesa el beneficio del otro, donde darse es fuerza motriz para la creatividad, la innovación, la responsabilidad, la madurez personal, y, como consecuencia de ello, la madurez de toda la organización.

Lo dicho hasta aquí se ajusta perfectamente a la tendencia que sugiere la perspectiva humanista de la nueva sensibilidad expuesta por Llano (1989, pp.154-155) cuando se refiere a los "valores ascendentes en la cultura de la empresa":

Las finalidades de la empresa son cuatro: a) proporcionar un servicio a la comunidad social; b) generar un suficiente valor económico añadido (beneficio); c) proporcionar a sus miembros satisfacción personal y perfeccionamiento humano, y d) lograr una capacidad de autocontinuidad o permanencia.

\section{A modo de conclusión}

Hoy por hoy, las personas están sometidas a estímulos constantes a través de la publicidad, los medios masivos, las redes sociales, por lo que captar su atención se torna un desafío cada vez más arduo. Lograr reducir el esfuerzo del cliente y generar experiencias memorables en los encuentros de servicio se convierte, por consiguiente, en una labor fundamental, a nivel estratégico, dentro de las organizaciones. Se entiende que sin clientes fieles y vinculados, la visión de largo plazo de las empresas se convierte en una utopía. Luego de desarrollar el concepto de experiencia entre un cliente y un encuentro de servicio, se comprende que para que sea recordada positivamente, la experiencia debe incluir elementos sensoriales, emocionales y tangibles que permitan que esto sea posible. Así, la generación de experiencias no se da al azar, sino que, por el contrario, es un proceso que implica planificación, organización, dirección y control.

Los grandes protagonistas en esta misión son las personas involucradas, visible o no visiblemente, en cada momento de la verdad. Por este motivo, contar con una cultura organizacional orientada a la generación de experiencias, y no al mero cumplimiento de la tarea, se torna crítico. Crear esta cultura orientada al servicio implica alinear a sus miembros en un objetivo común, propio de todos, integrado en su actuar y con real sentido: el servir a otro. Se puede decir que lo anterior resulta un gran desafío, pero a su vez, un objetivo 
trascendente, que enriquece a las personas y las hace más íntegras en su ser y en su actuar.

Tocar temas de la relación e importancia de las personas en la entrega de servicio, generando "culturas de servicio" desde un esquema de "liderazgo servidor" se tornan cruciales para lograr estructurar la manera de hacer negocios en la actualidad. Si bien probar esta hipótesis empíricamente requiere insertarse profundamente en organizaciones que velen por este fin, conocer su estructura sistémica, involucrarse en los procesos y gestión de personas, existen casos citados anteriormente en este trabajo (por ejemplo, Almacenes Sears, NCR, Ryder Truck, 30 Bancos del Oeste Medio de los Estados Unidos), donde se observa que el concepto de "espejo de satisfacción" es real. Es decir, que hay una relación inherente y directa entre la satisfacción de los empleados y la satisfacción de los clientes, lo cual genera comportamientos de lealtad en ellos, y la consecuente rentabilidad y crecimiento de la organización. La satisfacción del empleado comienza por la "calidad del servicio interno", que abarca el diseño del trabajo y de su lugar, la selección y desarrollo de las personas en esos puestos, las recompensas y reconocimiento y las herramientas para servir a los clientes, dispuesto en el modelo de la "cadena de rentabilidad del servicio", o Service Profit Chain, y forja la retención y productividad de las personas. Esto, a su vez, se traduce en la generación de una mejor "calidad de servicio externo", y, por consiguiente, en la satisfacción y lealtad de los clientes (Heskett et al., 1994).

No es utópico pensar que un valor como el servicio pueda ser usado positivamente para generar una verdadera mentalidad de concordia, de aprendizaje y de estimulantes experiencias entre el cliente y el proveedor. Lo utópico sería no intentarlo. En un mundo competitivo donde el libre mercado exige creatividad y rapidez para captar clientes y necesidades insatisfechas, es necesario re-inventar el modo como se relaciona con los clientes. Muchas veces se "utiliza" al cliente, se le manipula para que caiga en los lazos de una relación en la que pareciera que lo único importante es el intercambio de mercancías sin que importe nada más. Para superar esta realidad es preciso rescatar valores nobles y elevados como el auténtico servicio.

Se requiere, por tanto, construir y hacer vivir una verdadera filosofía de servicio dentro de las organizaciones, pues esta es "la raíz de la cultura organizacional", y abandonar con ello la creencia de que el servicio se vive solo a través de la implementación de procesos, y establecer en la organización la importancia de anidar desde los principios estratégicos la noble causa de "servir a otros".

Lo que las empresas hacen, permanentemente, es brindar a los seres humanos la oportunidad de trabajar, y de alguna manera implícita, servir a la sociedad. Y al trabajar se pueden captar dos planos que enriquecen la actividad laboral: todo trabajo humano tiene un plano objetivo y un plano subjetivo. Sobre lo objetivo podemos aseverar que todo trabajo transforma el mundo, produce riqueza y bienestar, y es espacio de relaciones con otras personas. En síntesis, el trabajo objetivo es lo tangible, lo medible, lo palpable. Pero, a la vez, todo trabajo es subjetivo porque no solo transforma el mundo real adaptándolo a las necesidades humanas, sino que quien trabaja se realiza a sí mismo como ser humano, crece como ser humano, y, en cierto sentido, se hace más ser humano (Burgos, 2003).

Quien sirve al cliente externo y al cliente interno origina un bienestar tangible que es retribuido. Por ende, hay una ganancia. Hablamos del plano objetivo del trabajo. Pero cuando un miembro de la organización ofrece un servicio desinteresado, generoso, esa acción cambia, modifica, enriquece interiormente a quien ofrece ese servicio. Estamos hablando del plano subjetivo. Con esa disposición genuina, cada trabajador "forja su carácter, desarrolla virtudes y aptitudes específicas, aprende a convivir y a cooperar con los demás, contribuye al bienestar de la sociedad, etc." (Burgos, 2003, p. 267).

El servicio al cliente interno como principio potenciador de la organización -se puede concluir- influye de manera contundente en estas dimensiones que se acaban de explicar, pero sobre todo brinda el equilibrio necesario: lo objetivo no está sobre lo subjetivo, ni lo subjetivo sobre lo objetivo. Hay que producir y generar riqueza, pero es preciso asegurar que cada trabajador aprenda a ser mejor persona sirviendo a los demás -al cliente externo y al interno- de modo genuino.

\section{REFERENCIAS}

Albrecht, K. (1992). Servicio al cliente interno. Barcelona: Paidós.

Aristóteles (1973). Ética Nicomaquea. México D. F.: Porrúa, S.A.

Burgos, J. M. (2003) Antropología: una guía para la existencia. Madrid: Palabra. 
Carlzon, J. (1991). El momento de la verdad. Madrid: Díaz de Santos.

Drucker, P. (1981). Gestión dinámica. Barcelona: Hispano Europea S.A.

Ehrhart, K. H., Witt, L. A., Scheider, B., \& Perry, S. J. (2011). Service Employees Give as They Get: Internal Service as a Moderator of the Service Climate-Service Outcomes Link. Journal of Applied Psychology, 96(2), 423-431.

Frankl, V. (1984). La idea psicológica del hombre. Madrid: Rialp.

Ginebra, J. \& Arana de la Garza, R. (1991). Dirección por servicio. México D. F.: McGraw-Hill.

Graterón, L. (1995). Curso de capacitación directiva. Trabajo no publicado. Luis Graterón y Asociados, Caracas.

Hellriegel, D. \& Slocum, J. W. (2009). Comportamiento organizacional. México D.F.: Cengage Learning.

Heskett, J., Jones, T., Loveman, G., Sasser, W., \& Schlesinger, L. (1994). Putting the Service-Profit Chain to Work. Harvard Business Review, 72(2), 164-174.

Horovitz, J. (1990). La calidad del servicio. Madrid: McGraw-Hill.

Juran, J. M. (1990). Juran y el liderazgo para la calidad. Madrid: Díaz de Santos S.A.

Lassey, W., \& Marshall, S. (1988). Leadership and Social Change. San Diego: University Asociates, Inc.

Leal, A. \& Valle, R. (1989). Los valores culturales y el diseño de las organizaciones andaluzas en el horizonte del año 2000. Revista de Economía y Empresa, 9(23), 21-36.

Lescano, L. (2012). Líderes de servicio. Madrid: Ediciones Internacionales Universitarias.

Liao, H., Toya, K., Lepak, D., \& Hong, Y. (2009). Do They See Eye to Eye? Management and Employee Perspectives of High-Performance Work Systems and Influence Processes on Service Quality. Journal of Applied Psychology, 94(2), 371-391.

Llano, A. (1989). La nueva sensibilidad. Madrid: Espasa Calpe.

Lovelock, C., Reynoso, J., D’Andrea, G., Huete, L. \& Wirtz, J. (2011). Administración de Servicios. Estrategias para la creación de valor en el nuevo paradigma de los negocios. México D.F.: Pearson.

Mattsson, J. (1994). Using Service Process Models to Improve Service Quality. En Managing Service Quality, 4(1), 47-52.
Melé Carné, D. (1992). Empresa y economía al servicio del hombre. Mensajes de Juan Pablo II a los empresarios y directivos económicos. Pamplona: EUNSA.

Morras, R., \& Polizzi, P. (2002). Gestión de la experiencia: El nuevo paradigma para crear valor. Trabajo no publicado. Universidad Adolfo Ibáñez, Santiago.

Normann, R. (1989). La gestión en las empresas de servicio. Bilbao: Deusto.

Pérez López, J. (2014). Fundamentos de la dirección de empresas. Madrid: Rialp.

Pine, J., \& Gilmore, J. (1998, julio-agosto). Welcome to the Experience Economy. Harvard Business Review, 97-105.

Real Academia Española (2014). Tomo I (23a‥ ed.). Madrid: Espasa Libros y Grupo Editorial Planeta.

Robbins, S. P. \& Judge, T. (2013). Comportamiento organizacional. México D.F.: Pearson.

Rosander, A. (1992). La búsqueda de la calidad en los servicios. Madrid: Díaz de Santos, S.A.

Schneider, B. \& Bowen, D. (1993). Los recursos humanos en la calidad del servicio. Harvard Deusto Business Review, 57, 68-67.

Senlle, A. (1993). Calidad total en los servicios y en la administración pública. Barcelona: Gestión 2000, S.A.

Scholtes, P. (1991) El manual del equipo. Madison: Joiner Associates Incorporated.

Shaw, J. (1991). Gestión de servicios. Madrid: Díaz de Santos, S.A.

Temple, R., \& Droege, R. (1994). Internal Customers Need Delighting Too. Managing Service Quality, 4(1), 14-17.

Thornely, N., \& Lees, D. (1993). Leadership: The Art of Motivation. London: Century Business.

Zeithaml, V., Bitner, M. \& Gremler, D. (2009). Marketing de Servicios. México D.F.: McGraw-Hill.

Zeithaml, V., Parasuraman A. \& Berry, L. (1993). Calidad total en la gestión de servicios. Madrid: Díaz de Santos, S.A.

Zimmermann, C. \& Enell, J. (1993). Empresas de servicio. En Juran et al., Manual de control de calidad. (Vol. II). Madrid: McGraw-Hill.

Zomerdijk, L., \& Voss, C. (2010). Service Design for Experience-Centric Services. Journal of Service Research, 13(1), 67-82. 